\section{$\underset{\substack{\text { hommes } \\ \text { \& migrations }}}{ }$}

\section{Hommes \& migrations}

Revue française de référence sur les dynamiques

migratoires

$1327 \mid 2019$

Capitales européennes et diversité culturelle

\title{
Revues Plurielles : « Les écritures en exil et de l'exil » au salon LittExil
}

\section{Yannick Kéravec}

\section{(2) OpenEdition \\ Journals}

\section{Édition électronique}

URL : https://journals.openedition.org/hommesmigrations/10283

DOI : 10.4000/hommesmigrations. 10283

ISSN : 2262-3353

Éditeur

Musée national de l'histoire de l'immigration

\section{Édition imprimée}

Date de publication : 1 octobre 2019

Pagination : 136-140

ISBN : 978-2-919040-47-6

ISSN : $1142-852 X$

\section{Référence électronique}

Yannick Kéravec, «Revues Plurielles : «Les écritures en exil et de l'exil » au salon LittExil », Hommes \& migrations [En ligne], 1327 | 2019, mis en ligne le 01 octobre 2019, consulté le 06 janvier 2022. URL http://journals.openedition.org/hommesmigrations/10283; DOI : https://doi.org/10.4000/ hommesmigrations. 10283 


\title{
LHTHERATURE
}

\section{Revues Plurielles: «Les écritures en exil et de l'exil» au salon LittExil}

\author{
Yannick Kéravec, \\ Ent'Revues.
}

Que ce soient des parcours personnels ou des traversées collectives, les œuvres littéraires font (re)vivre l'exil, parfois avec l'épaisseur du passé ou en résonnance avec l'actualité la plus brûlante. Placées sous le signe de la diversité, les revues dessinent des espaces où les écritures de l'exil dialoguent avec les écritures en exil. Elles diffusent ces odyssées modernes qui sont aussi des tentatives d'expérimenter des nouvelles écritures de l'intime.

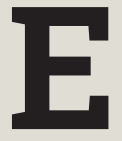

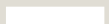

nt'revues, le site des revues culturelles, s'est associé à la troisième édition du salon LittExil en réunissant des éditeurs et des revuistes qui ont débattu sur les questions de migrations, d'exil, de mémoire, interrogeant ainsi les évolutions éditoriales des revues et leurs conséquences. En guise d'ouverture, Yannick Kéravec (Ent'revues) a donné la parole à cinq revues - À littérature-action, Riveneuve Continents, Exils et migrations ibériques au XX $X^{e}$ siècle, Rue Saint-Ambroise, Gibraltar - qui, « placées sous le signe de la diversité, dessinent des espaces où les écritures de l'exil dialoguent avec les écritures en exil (...) qui diffusent ces odyssées modernes qui sont aussi des tentatives d'expérimenter des nouvelles écritures de l'intime».

\section{Premier panorama des revues sur l'exil}

La table ronde a permis de faire un panorama de certaines revues francophones qui se sont présentées en précisant leur ligne éditoriale. Première à s'exprimer, Marie Virolle a dédié son intervention à Aziz Chouaki décédé le 16 avril dernier. Connu pour son roman L'Étoile d'Alger (2002), il fut publié dans un des premiers numéros de la revue. À littérature-action a été créée en 1996 sous l'enseigne des éditions Marsa. Elle s'est intitulée Algérie Littérature/Action jusqu'en 2017. Fondée durant la décennie noire, quand toute l'édition fut sinistrée, et quand les écrivaines sont mort-es, exilé-es ou encore clandestin-es, elle se définit comme une revue « de l'exil, d'exil, en exil ». Lancée à Paris, ses contenus se concevaient et se publiaient sur «les deux rives ». Aujourd'hui, l'intérêt pour l'Algérie s'estompe. Les auteurs et autrices partent vers d'autres maisons d'édition ou d'autres projets. D'où l'ouverture sur d'autres horizons car « la migration [a] saisi quasi tout le 
continent africain ». Elle change de titre, garde son format et s'ouvre à d'autres langues, terroirs et pays. C'est le Tout-monde cher à Édouard Glissant, portant les idées de métissage, de créationmonde, de créolisation. Si «les idées autour des migrations circulent aisément dans les milieux parisiens, c'est moins évident en province ». Son implantation actuelle à Limoges, pôle de la francophonie, concrétise et rend ce glissement éditorial plus pertinent. À littérature-action croise les littératures migrantes ou enracinées, les imaginaires, tous les types d'écriture: prose, poésie, théâtre...; elle ne se veut pas multiculturelle, ou interculturelle, mais bien transculturelle : c'est plus profond encore.

Riveneuve Continents est représentée par Gilles Kraemer, Patrick Navaï et Reza Afchar Naderi. À l'origine, Riveneuve Continents est une maison d'édition de Marseille, créée par des gens "ouverts sur le monde», des traducteurs, publiant des

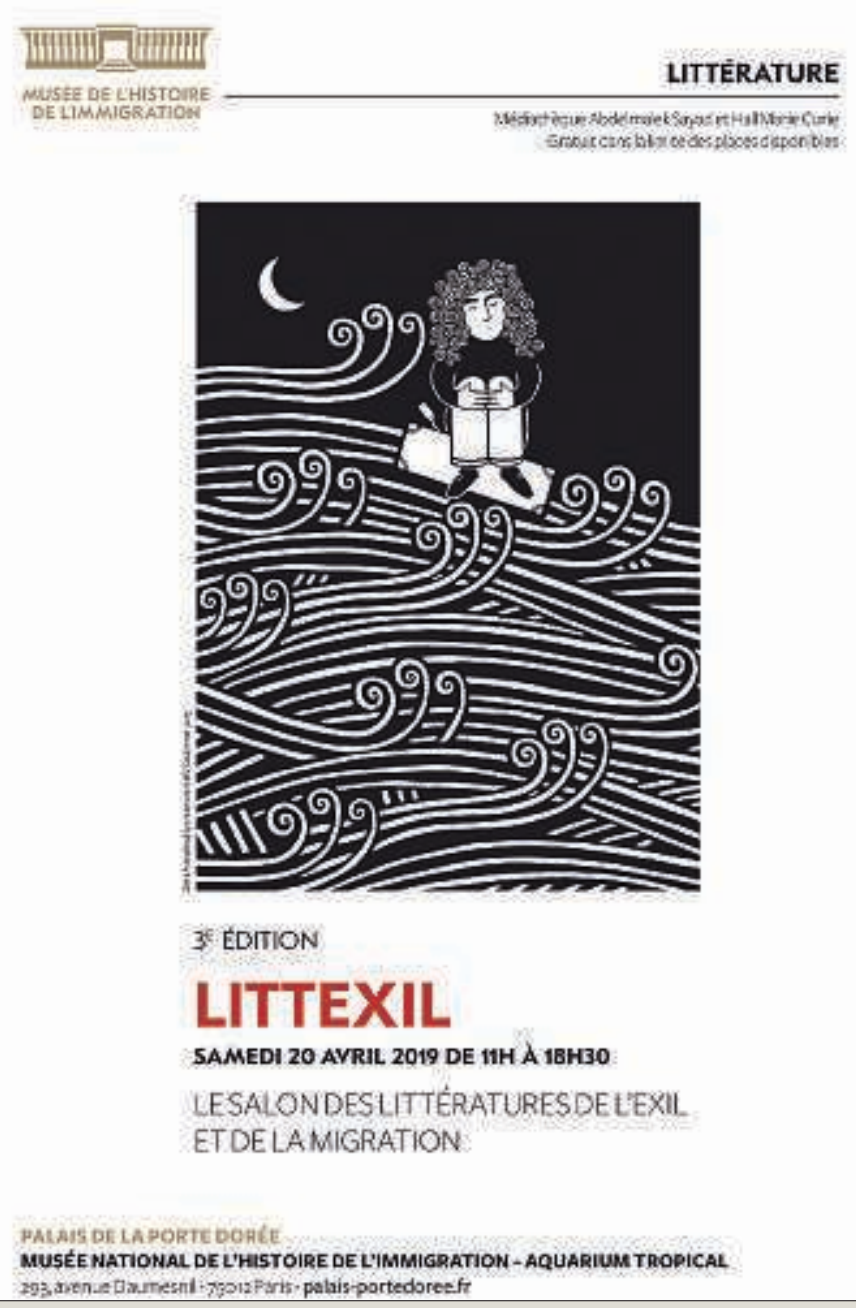

- Affiche du Salon LittExil, Paris, avril 2019.

(C) Sandy Chamaillard, Palais de la Porte Dorée textes courts et inédits d'auteurs connus ou inconnus, au départ de Scandinavie et du Sud; elle se dote d'une revue semestrielle pour accueillir des textes courts et ainsi créer une communauté éditoriale. Chaque numéro est dédié à un pays, à une zone géographique francophone: Tunisie, Vietnam, Haïti après la catastrophe, les « décolonisations littéraires » en Afrique, etc. La revue s'ouvre à de nombreuses thématiques. Celle des "rives» revient de manière structurante, parfois même sur tout un numéro, comme celui sur les diasporas de la Tunisie «l'ici, là-bas et entre les deux rives ${ }^{1}$ ». La migration peut aussi être un exil intérieur, celui de l'écrivain qui se sent en décalage avec son environnement. «Être et se sentir exilé, c'est un peu la même chose. 》 Gilles Kraemer mentionne, parmi les territoires abordés, le roman - l'autofiction -, l'égo-histoire, l'égo-sociologie, mais aussi le cinéma, le théâtre, la danse, qui 
traitent cette dimension d'éloignement et d'exil. Selon un montage peu habituel, le second numéro semestriel de Riveneuve Continents accueille depuis 2013 la revue Exils et migrations ibériques au $\mathrm{XX}^{e}$ siècle, élaborée par le Cermi (Centre d'études et de recherche sur les migrations ibériques).

La revue Rue Saint-Ambroise présentée par Bernardo Toro, son fondateur, existe depuis 20 ans. Elle a été créée pour valoriser l'essor des nouvelles littéraires en France et d'une nouvelle génération d'auteurs. Beaucoup de jeunes auteurs et autrices publient sur Internet, sous des formes d'autoédition. La nouvelle a toujours eu en France une place discrète pour des raisons sociologiques, économiques, politiques, éditoriales, historiques... mais la vraie raison est littéraire. Cette forme est morte, si elle n'est plus capable de «nous raconter en 2019 ». « Si [la nouvelle] va mal, toute la littérature va mal. » Rue Saint-Ambroise est aussi une maison d'édition toute jeune qui a coédité, avec le Musée national de l'histoire de l'immigration, un recueil de nouvelles sur Paris à partir du regard des migrants où l'on trouve de multiples pays et continents.

Santiago Mendieta présente la revue Gibraltar créée en 2012. Parution annuelle de récits, de reportages, c'est une « revue de combat sans être militante » proposant une grande diversité de formes: des textes à la photo, des reportages à la fiction... Elle incarne le «slow journalism » dans la foulée de la revue XXI (mook créé en 2008): «On prend le temps, on raconte les choses sur la longueur et on fait une place à la fiction. » Tous les numéros publient une nouvelle, un récit original... La revue est toujours dédiée aux rives et aux mondes méditerranéens, foisonnants, en guerre, souffrant de problèmes sociaux, économiques, marqués par les migrations, les exils, la guerre en Syrie, les tragédies... Gibraltar est ainsi le reflet de ces mondes écartelés. Sa périodicité annuelle offre le temps d'explorer un thème et d'observer les situations inhabituelles en s'écartant des sujets à la mode.

\section{Exploration du thème de l'exil dans les revues}

Dans un deuxième temps, les revues ont pu élargir leurs réflexions en se concentrant sur leurs numéros en cours. La revue À littérature-action stipule que «tout écrivain est un exilé ». Marie Virolle revient sur le récit tragique de l'errance d'un jeune journaliste guinéen, réfugié politique, que la revue a aidé dans sa demande d'asile pour "passer des paroles aux actes». Elle présente Dernier nid pour les cigognes, roman-récit de déracinements, d'enracinements, du Chili à l'Argentine, concernant plusieurs générations et continents, sur la mémoire et le mystère de la mort du fils. La revue s'est élargie de la littérature aux arts plastiques et graphiques. Ainsi, le numéro sur Le Caravage développe l'idée de la circulation et de la transculturalité d'une période antérieure, la Renaissance, qui a connu une interpénétration des arts. Le «transculturel» s'illustre aussi dans le numéro sur le parcours d'Albert Cossery, artiste, chrétien, syrien d'origine mais ayant vécu en Égypte, exilé en France, à Paris où il logea dans une petite chambre de l'hôtel La Louisiane dans le Quartier latin, de 1945 à 2008. Ses écrits parlent beaucoup de l'Égypte et mêlent onirisme et réalisme. Dans un prochain numéro, «Création et migration. Apartheid des gens 
apatrides 》, titre du colloque organisé par l'université de Limoges en 2019, la revue abordera le thème «Création et migration 》. Si tout écrivain est un exilé, ses parcours produisent des fictions à tous les niveaux. «La littérature française se régénère par ses métèques 》 : d'où la nécessité d'une créativité langagière ou thématique, dans le métissage des imaginaires autour de la littérature de l'exil.

Pour la revue Riveneuve Continents, Gilles Kraemer évoque le numéro « Algérie : la nahda des Lettres, la renaissance des mots», paru au printemps 2015, qui a annoncé la situation d'aujourd'hui. Il mentionne un autre numéro sur l'exil : « Les nouvelles lettres persanes » que présente Reza Afchar Naderi, iranien, poète et traducteur. Ce dernier revient sur l'autre « genre oublié en France : la poésie ». La France a besoin de poètes qui viennent d'ailleurs pour redonner vie à un genre défaillant. Le media Slate signale que la poésie représente seulement $0,3 \%$ du marché de l'édition en France. Au contraire, en Iran, c'est un « art majeur » parlé et pratiqué par tous et toutes, depuis l'homme de la rue au plus haut niveau de l'État, du berceau à la tombe. Tout le monde peut réciter de la poésie classique. Hafez, poète le plus populaire, écrivait au XIV siècle. Si la poésie est florissante en Iran, en France, elle résonne aujourd'hui dans un grand vide. Yannick Kéravec évoque cependant le Marché de la poésie de Paris qui se déroule chaque année en juin (avec un kiosque de revues confié à Ent'revues), et un nouveau salon de revues de poésie dont une édition a été confirmée à Marseille...

Emma Rubio-Milet, auteure et membre du comité scientifique, présente le dernier numéro d'Exils

(र La migration peut aussi être un exil intérieur, celui de l'écrivain qui se sent en décalage avec son environnement.

et migrations ibériques au XIX siècle ( $\mathrm{n}^{\circ}$ 9-10, hiver 2018-été 2019), consacré aux «Écritures de la révolution et de la guerre d'Espagne 》. Cette revue a été créée en 1993, mais une nouvelle série débute en 2004 par la publication de « Témoignages d'exils entre parole et silence: regards et points de vue ». En 2018, son thème était « Comment voyagent les souvenirs des enfants d'exilées? », à partir des témoignages d'enfants d'immigrés espagnol-es, récits fondés sur une expérience historique transmise et de fait interprétée. Ces expériences de vie sont représentées sous différentes formes, des récits, des écrits ou des productions plus élaborées : les archives privées et les témoignages permettent de créer des nouvelles, des documentaires, des reportages audio ou vidéo... Des analyses scientifiques croisées offrent une vraie polyphonie critique. Le dossier est illustré d'une riche iconographie, issue d'archives et de reproductions d'artistes espagnols. Ces récits, prenant source il y a 80 ans, restent d'actualité aujourd'hui, notamment par la question politique de l'accueil.

La publication d'un recueil de nouvelles par la maison d'édition Rue Saint-Ambroise avec le MNHI s'est faite à l'occasion d'une résidence d'écriture au Musée. C'est le fruit d'une collaboration avec la revue Hommes \& Migrations de plus de dix ans qui a abouti à ce résultat. La démarche éditoriale est simple : 10 auteurs rencontrent 10 migrants, les migrants racontent leur expérience et les auteurs ne transcrivent pas de manière exhaustive mais en font une fiction, intègrent 


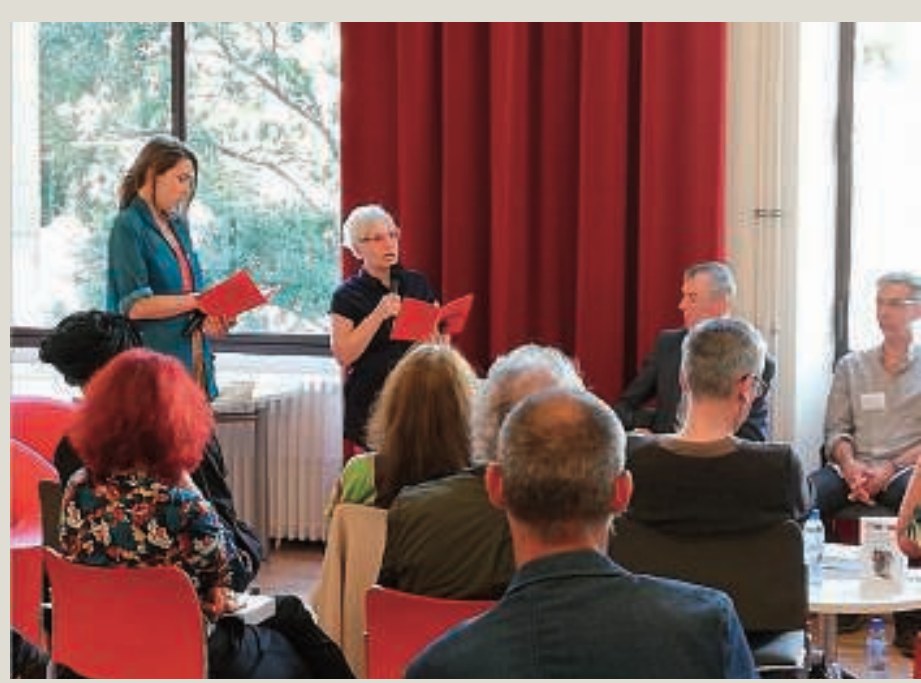

- Conférence salon LittExil, Paris, avril 2019. Photo Charlotte Perdriau. (c) Charlotte Perdriau, Musée national de l'histoire de l'immigration des éléments dans une création. La littérature est considérée ici comme un moyen de connaissance. Certaines histoires personnelles échappent en effet aux approches scientifiques: sociologie, anthropologie, histoire... Si une grande part de la littérature est constituée par du témoignage romancé, du témoignage que l'on particularise, il existe peu de véritable littérature d'exil.

Santiago Mendieta, de la revue Gibraltar, présente un reportage sur un village d'Andalousie qui expérimente des formes de militance, d'auto-construction, d'utopie... sous les mandats du maire, réélu depuis 1979. Cet article est le fruit d'une immersion de 10 jours sur place. Dans le numéro en cours, dans la partie «Fiction》, la rédaction a prétendu avoir redécouvert le journal d'exil d'Ovide, en réalité une création présentée comme un véritable document historique - avec beaucoup de notes. C'est une manière de créer un pont entre la nouvelle, la littérature, les témoignages et la poésie syrienne. La revue est généraliste mais fait appel à des auteurs spécialistes dans leurs domaines. Le numéro comporte toujours un dossier avec trois, quatre, cinq sujets. Ici, «Les voix de la liberté » rejoignent la philosophie : l'étape ultime de Port-Bou pour Walter Benjamin et son destin tragique en exil : classé comme apatride, indésirable, il ne parviendra pas à quitter la France. Aux antipodes, on retrouve un personnage de Leucate, Henri de Monfreid, pirate, flibustier, romancier. Cela permet de donner la parole à différents poètes-ses exilé-es contemporain-es. En complément des textes et des témoignages, la revue publie une bande dessinée sur les fosses du franquisme en Espagne et des récits-photos décrivant le parcours d'une famille syrienne. Portant du particulier, de l'intime, la revue tend ainsi vers l'universel.. • 